\section{CD47XPD-L1 BISPECIFIC ANTIBODIES FOR CANCER THERAPY}

${ }^{1}$ Xavier Chauchet*, ${ }^{1}$ Elise Pernarrieta, ${ }^{1}$ Nicolas Bosson, ${ }^{1}$ Sébastien Calloud, ${ }^{1}$ Louis Hellequin, ${ }^{1}$ Margaux Legrand, ${ }^{1}$ Alizée Viandier, ${ }^{1}$ Françoise Richard, 'Laura Cons, ${ }^{1}$ Pauline Malinge, 'Tereza Bautzova, 'Jérémie Bourguignon, 'Guillemette Pontini, ${ }^{2}$ Mengzhu Sun, ${ }^{1}$ Ulla Ravn, ${ }^{1}$ Valéry Moine, ${ }^{1} Y$ ves Poitevin, ${ }^{2}$ Stéphanie Hugues, ${ }^{1}$ Nicolas Fischer, 'Limin Shang, 'Walter Ferlin, 'Krzysztof Masternak. 'Light Chain Bioscience/Novimmune, Plan les Ouates, Switzerland; 'University Medical Center (CMU), Geneva, Switzerland

Background PD-1/PD-L1 blockade can significantly improve survival across many types of cancer, but only in a minority of patients. To broaden its therapeutic efficacy, several combination partners are now being evaluated together with PD-1/ PD-L1 blockade. Agents blocking CD47/SIRP $\alpha$ innate immune checkpoint are one such example, and co-targeting PD-1/PDL1 and CD47 with monoclonal antibody (mAb) combinations showed increased antitumor responses in preclinical studies. However, CD47 mAbs are hindered by ubiquitous CD47 expression leading to rapid target-mediated clearance and safety concerns. Consequently, dual-targeting CD47xPD-L1 bispecific antibodies (bsAbs) enabling preferential inhibition of CD47 on PD-L1-positive cells are being tested as an alternative approach. We compare here two distinct bsAbs, based on a common PD-L1 antibody arm, with differing FcgR-enabling effector functions and CD47-binding arm affinities.

Methods An array of fully human bsAbs associating a high affinity PD-L1 arm to CD47 arms with varying affinities were generated using the $\kappa \lambda$-body platform. ${ }^{1}$ CD47xPD-L1 bsAbs of human IgG1 isotype (CD47 low affinities) or IgG4 isotype (CD47 high affinities) were screened in various binding assays (including to red blood cells $(\mathrm{RBC})$ ) and in receptor-blocking assays, and then tested for their Fc-mediated killing and T-cell activation activity (SEA-stimulated PBMC assay). Selected molecules were evaluated in vivo.

Results Both bsAb approaches demonstrated strong blockade of PD-1/PD-L1 interaction and significantly enhanced T-cell activation in vitro. CD47lowxPD-L1 IgG1 bsAbs did not bind to $\mathrm{RBC}$ and showed PD-L1-guided inhibition of CD47. ADCP and ADCC experiments with a panel of tumor cell lines expressing various target levels showed superior killing activity with CD47lowxPD-L1 IgG1 bsAbs as compared to the antiPD-L1 IgG1 mAb, avelumab. On the other hand, CD47highxPD-L1 IgG4 bsAbs showed residual RBC binding and PD-L1-independent blocking of CD47/SIRP $\alpha$ These CD47high IgG4 bsAbs were able to enhance the anti-tumor activity of anti-tumor-associated antigen (TAA) mAbs in vitro (phagocytosis), and in vivo (Raji lymphoma xenograft model). In addition, anti-tumor activity of mouse CD47xPD-L1 bsAbs in a syngeneic MC38 colon carcinoma model was demonstrated.

Conclusions With the objective of finding the optimal CD47xPD-L1 bsAb design, two approaches targeting CD47 and PD-L1 inhibition were tested. Both the CD47lowxPD-L1 IgG1 bsAbs and CD47highxPD-L1 IgG4 bsAbs were able to mediate enhanced antitumor responses, the former as a standalone treatment, the latter in conjunction with an anti-TAA mAb. To further characterize the CD47lowxPD-L1 and CD47highxPD-L1 bsAbs, lead candidates will be tested in PK and tolerability studies in non-human primates.

\section{REFERENCES}

1.. Fischer N, Elson G, Magistrelli $G$, Dheilly $E$, Fouque $N$, Laurendon $A$, et al. Exploiting light chains for the scalable generation and platform purification of native human bispecific IgG. Nat Commun 2015 May;6(1):6113.

http://dx.doi.org/10.1136/jitc-2021-SITC2021.265 